\title{
Searching for the optimal stimulus eliciting auditory brainstem responses in humans
}

\author{
Fobel, Oliver; Dau, Torsten
}

Published in:

Journal of the Acoustical Society of America

Link to article, DOI:

$10.1121 / 1.1787523$

Publication date:

2004

Document Version

Publisher's PDF, also known as Version of record

Link back to DTU Orbit

Citation (APA):

Fobel, O., \& Dau, T. (2004). Searching for the optimal stimulus eliciting auditory brainstem responses in humans. Journal of the Acoustical Society of America, 116(4), 2213-2222. https://doi.org/10.1121/1.1787523

\section{General rights}

Copyright and moral rights for the publications made accessible in the public portal are retained by the authors and/or other copyright owners and it is a condition of accessing publications that users recognise and abide by the legal requirements associated with these rights.

- Users may download and print one copy of any publication from the public portal for the purpose of private study or research.

- You may not further distribute the material or use it for any profit-making activity or commercial gain

- You may freely distribute the URL identifying the publication in the public portal

If you believe that this document breaches copyright please contact us providing details, and we will remove access to the work immediately and investigate your claim. 


\title{
Searching for the optimal stimulus eliciting auditory brainstem responses in humans
}

\author{
Oliver Fobel ${ }^{\mathrm{a})}$ \\ Medizinische Physik, Carl von Ossietzky Universität Oldenburg, D-26111 Oldenburg, Germany \\ Torsten Dau ${ }^{\text {b) }}$ \\ Centre for Applied Hearing Research, Orsted-DTU, Technical University of Denmark, DK-2800 Kgs. \\ Lyngby, Denmark
}

(Received 8 August 2003; revised 29 June 2004; accepted 6 July 2004)

\begin{abstract}
This study examines auditory brainstem responses (ABR) elicited by rising frequency chirps. Two chirp stimuli were developed and designed such as to compensate for cochlear travel-time differences across frequency, in order to maximize neural synchrony. One chirp, referred to as the O-chirp, was based on estimates of human basilar membrane (BM) group delays derived from stimulus-frequency otoacoustic emissions (SFOAE) at a sound pressure level of $40 \mathrm{~dB}$ [Shera and Guinan, in Recent Developments in Auditory Mechanics (2000)]. The other chirp, referred to as the A-chirp, was derived from latency functions fitted to tone-burst-evoked ABR wave-V data over a wide range of stimulus levels and frequencies [Neely et al., J. Acoust. Soc. Am. 83(2), 652-656 (1988)]. In this case, a set of level-dependent chirps was generated. The chirp-evoked responses, particularly wave- $\mathrm{V}$ amplitude and latency, were compared to click responses and to responses obtained with the original chirp as defined in Dau et al. [J. Acoust. Soc. Am. 107(3), 1530-1540 (2000)], referred to here as the M-chirp since it is based on a (linear) cochlea model. The main hypothesis was that, at low and medium stimulation levels, the O- and A-chirps might produce a larger response than the original M-chirp whose parameters were essentially derived from high-level BM data. The main results of the present study are as follows: (i) All chirps evoked a larger wave-V amplitude than the click stimulus indicating that for the chirps a broader range of spectral components contributes effectively to the ABR. (ii) Only small differences were found between the O-chirp and M-chirp responses at low and medium levels. This indicates that SFOAE may not provide a robust estimate of BM group delay, particularly at low frequencies, or that frequency-dependent neural delays exist which are not reflected in the design of these chirps. (iii) The A-chirp produced the largest responses, particularly at low stimulation levels. This chirp might therefore be valuable for clinical applications, particularly in tests where the click stimulus has been used so far. (C) 2004 Acoustical Society of America.
\end{abstract}

[DOI: 10.1121/1.1787523] [S0001-4966(94)70510-5]

PACS numbers: 43.64.Qh, 43.64.Ri, 43.64.Bt [WPS] Pages: 2213-2222

\section{INTRODUCTION}

Transient stimuli like clicks are commonly used in electrophysiological research of the human auditory system to elicit synchronized auditory brainstem responses (ABR). The click is the most common stimulus used in recording the $\mathrm{ABR}$, whether for neurodiagnostic or audiologic purposes. However, in the cochlea, the response to a click is not entirely synchronous: The peak of the response occurs several milliseconds later in low-frequency channels than it does in high-frequency channels (e.g., von Békésy, 1960; Kiang et al., 1965; Kiang, 1975). This is mainly a result of the change of stiffness along the cochlear partition. As a consequence, the phase velocity of the traveling wave depends in a characteristic way upon location, which causes spatial dispersion. It takes more time for the low-frequency region to

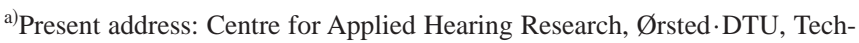
nical University of Denmark, DK-2800 Kgs. Lyngby, Denmark; electronic mail: of@oersted.dtu.dk

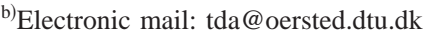

reach maximal displacement at the apical end of the cochlea. Electrophysiological responses to broadband transients like clicks appear to be largely generated by the synchronized activity of the high-frequency channels on their own. For example, Don and Eggermont (1978) measured human ABR in response to clicks masked by high-pass noise with different cut-off frequencies. This masking technique revealed that the latencies in response to low-frequency stimuli are delayed relative to high frequencies. Don and Eggermont concluded from their data that all frequency regions contribute to the ABR but that the response is dominated by contributions from the 2-3 octaves towards the basal end.

In a later study, Don et al. (1994) developed a technique of "normalizing" click-evoked ABR using high-pass noise masking. They adjusted for differences in the neural conduction time (wave $I-V$ delay) through compression or expansion, and for differences in the cochlear response time through shifts of the derived "narrowband" ABR patterns. A summation of the compressed and time shifted responses resulted in the so-called "stacked ABR." Their study demonstrated the effect of temporal delays of cochlear activation on 
the component amplitude of the compound ABR. In a later study, Don et al. (1997) showed that this technique is particularly useful for the detection of small intracanalicular acoustic tumors.

A different approach was used by Dau et al. (2000) and Wegner and Dau (2002) to study the effects of cochlear dispersion on the formation of ABR. They demonstrated that upward chirps can strongly affect ABR wave V. Their chirp was designed to produce simultaneous displacement maxima along the cochlear partition by compensating for frequencydependent traveling-time differences. Their equations determining the temporal course of the chirp were derived on the basis of a linear cochlea model (de Boer, 1980) and were calculated to be the inverse of the delay-line characteristic of the human cochlear partition. The fundamental relationship between stimulus frequency and place of maximum displacement was taken from Greenwood (1990). ABR evoked by the broadband chirp showed a larger wave-V amplitude than corresponding click-evoked responses. Dau et al. (2000) demonstrated that the ABR is not an electrophysiological event purely evoked by onset or offset of an acoustic stimulus, but that an appropriate temporal organization, determined in part by basilar-membrane (BM) traveling-wave properties, can significantly increase synchrony of neural discharges. Wegner and Dau (2002) demonstrated that such a chirp is also very useful for retrieving frequency-specific information, particularly at low frequencies.

The model of de Boer (1980) is based upon the experimental observations of von Békésy (1960). Von Békésy's measurements were performed with the aid of a microscope to detect and measure the movements of cochlear structures. The movements had to be much larger than under the influence of "natural" sounds. In fact, von Békésy used very high sound pressure levels (SPL), of the order of 120 to $140 \mathrm{~dB}$. In later studies, cochlear vibration patterns were measured with more sensitive techniques and under more natural circumstances. These studies of cochlear mechanics have established that the response of the BM to tones at characteristic frequency $(\mathrm{CF})$ is generally nonlinear and compressive while it responds essentially linearly to tones with a frequency well below CF (e.g., Rhode, 1971; Sellick et al., 1982; Robles et al., 1986; Ruggero et al., 1997; Rhode and Recio, 2000). At low stimulus levels, if plotted as isointensity curves as a function of stimulus frequency, the BM response patterns are sharply frequency tuned around the CF. Recent psychophysical studies suggest that the BM is also the primary source of the nonlinearities observed in masking experiments (e.g., Oxenham and Plack, 1998; Plack and Oxenham, 1998) and that cochlear nonlinearities have a significant influence on a wide range of basic auditory processes, such as frequency selectivity (e.g., Moore et al., 1999), temporal integration (Oxenham et al., 1997), and loudness growth (e.g., Yates et al., 1990; Moore and Glasberg, 1997). Damage to the cochlea, and in particular the outer hair cells, results in a reduction in sensitivity and a loss of compression at CF (Ruggero and Rich, 1991; Ruggero et al., 1995). In such a situation, the pattern of BM vibration is similar to the "insensitive" response, originally found by von Békésy.

Recently, Shera and Guinan $(2000,2003)$ introduced a method to measure BM group delay in the human auditory system. They measured stimulus-frequency otoacoustic emissions (SFOAE) for a stimulus level of $40 \mathrm{~dB}$ SPL, and derived the SFOAE group delay, $\tau_{\mathrm{SFOAE}}$, from the slope of the SFOAE-phase versus frequency function. According to the theory of coherent reflection filtering (Shera and Zweig, 1993; Zweig and Shera, 1995) the BM group delay $\tau_{\mathrm{BM}}$ is given by $\tau_{\mathrm{BM}}=0.5 \times \tau_{\mathrm{SFOAE}}$. It is assumed that, at low stimulus levels, the BM transfer functions can be described by minimum-phase-shift filters (Zweig, 1976). Shera et al. (2002) therefore directly related $\tau_{\mathrm{BM}}$ to cochlear-filter bandwidth. They compared the results to psychophysical estimates of auditory filter bandwidth obtained using notchednoise forward masking experiments (Shera et al., 2002; Oxenham and Shera, 2003). The estimates of frequency selectivity obtained in the different approaches were in good agreement. These estimates of cochlear frequency selectivity at low levels, obtained with the method of SFOAEs, will most likely differ from those values assumed in de Boer's (1980) model. Thus, a corresponding chirp stimulus that would be designed to compensate for frequency-dependent traveling-time differences, can be expected to differ in its waveform from the chirp developed by Dau et al. (2000).

Another approach to compensate for delays across frequency would be to base the chirp parameters on wave- $\mathrm{V}$ latency values obtained in tone-burst-evoked ABR data. Gorga et al. (1988) measured tone-burst-evoked ABRs over a wide range of stimulus levels and frequencies. Their waveV-latency data were described by Neely et al. (1988) by the following power-law relation:

$$
\tau_{b}=a+b c^{-i} f^{-d}
$$

where $i$ represents tone-burst intensity, $f$ indicates tone-burst frequency, and $a, b, c$, and $d$ are constants [cf. their Eq. (1)]. Neely et al. (1988) assumed that the first term, parameter $a$, represents the frequency and level-independent neural component of the latency while the second term in Eq. (1) reflects the mechanical component of the latency due to the propagation in the cochlea thus representing BM group delay. By comparing the ABR data from Gorga et al. (1988) with tone-burst otoacoustic emission(OAE) data from Norton and Neely (1987)—who used a subgroup of the subjects from Gorga et al. (1988) - Neely et al. (1988) found a much larger inter- and intraindividual variability in the OAE data than in the ABR data which might suggest that BM group delay can be better estimated with ABR than with OAE.

The current study deals with the development and test of chirp stimuli in an attempt to find an ideal stimulus eliciting ABR in humans. One chirp is generated based on the SFOAE data by Shera and Guinan (2000). The second chirp is generated on the basis of ABR wave- $\mathrm{V}$ latency data by Neely et al. (1988). Corresponding chirp-evoked responses are compared at various stimulus levels with results obtained with the original model-based chirp by Dau et al. (2000), and with conventional click data. The underlying hypothesis is that, at low stimulation levels, the new chirps might produce a better synchronization than the original chirp since the latter one was derived on the basis of high-level BM data. The 
results of the present study could be valuable both for a better understanding of basic mechanisms underlying the generation of ABR and for clinical applications using chirpevoked ABR as an objective indicator of hearing threshold.

\section{CHIRP STIMULI}

\section{A. OAE-based chirp stimulus (O-chirp)}

The first chirp stimulus is based on the experimental SFOAE data of Shera and Guinan (2000). They did experiments for stimulus frequencies in the range from 0.5 to 10 $\mathrm{kHz}$ in humans, at a level of $40 \mathrm{~dB}$ SPL. Emission group delays, $\tau_{\mathrm{SFOAE}}$, were calculated and related to BM group delays, $\tau_{\mathrm{BM}}=0.5 \times \tau_{\mathrm{SFOAE}}$, as a function of $\mathrm{CF}$. The data can be roughly approximated by the following function (Shera, personal communication):

$$
\tau_{\mathrm{BM}, \mathrm{O}}(f):=t(f)=c(f /[\mathrm{Hz}])^{\alpha},
$$

with the constants $c=0.15 \mathrm{~s}$ and $\alpha=-0.5 .{ }^{1} \tau_{\mathrm{BM}, \mathrm{O}}$ can also be interpreted as reflecting the propagation time $t(f)$ needed to arrive at the place of resonance for frequency $f$. In order to compensate for dispersion across frequency, we introduced the variable transformation $t \rightarrow t_{0}-t$, with $t_{0}$ $=\tau_{\mathrm{BM}, \mathrm{O}}(50 \mathrm{~Hz})$ and calculated the following inverse function $f_{\mathrm{O}}(t)=t^{-1}(f):^{2}$

$$
f_{\mathrm{O}}(t)=\left(\frac{c}{t_{0}-t}\right)^{2} \text {. }
$$

This function, reflecting the change of the instantaneous frequency with time was then integrated over time to derive the instantaneous phase $\varphi_{\mathrm{O}}(t)$ of the resulting chirp

$$
\begin{aligned}
\varphi_{\mathrm{O}}(t) & =2 \pi \int_{0}^{t} f_{\mathrm{O}}\left(t^{\prime}\right) d t^{\prime} \\
& =2 \pi c^{2}\left(\frac{1}{t_{0}-t}-\frac{1}{t_{0}}\right) .
\end{aligned}
$$

The chirp stimulus is then given by

$$
s_{\mathrm{O}}(t)=A_{\mathrm{O}}(t) \sin \left[\varphi_{\mathrm{O}}(t)-\varphi_{0}\right]
$$

whereby $\varphi_{0}$ determines the starting phase of the chirp. The amplitude factor $A_{\mathrm{O}}(t)$ was chosen as

$$
A_{\mathrm{O}}(t)=\sqrt{\frac{d f_{\mathrm{O}}(t)}{d t}}=\sqrt{\frac{2 c^{2}}{\left(t_{0}-t\right)^{3}}}
$$

in order to produce a stimulus with a flat magnitude spectrum. Since the stimulus $s_{\mathrm{O}}(t)$ is based on OAE data, it is referred to as the "O-chirp" throughout the present paper.

\section{B. ABR-based chirp stimulus (A-chirp)}

The second chirp stimulus developed in this study is based on the tone-burst-evoked ABR data by Gorga et al. (1988). They used tone bursts at ten frequencies $(0.25,0.5$, $0.75,1,1.5,2,3,4,6$, and $8 \mathrm{kHz}$ ) and nine intensities (20 to $100 \mathrm{~dB}$ SPL in 10-dB steps). Their data were described by Neely et al. (1988) by a power law [cf. Eq. (1)], relating latency to stimulus frequency, assuming that the total wave- $\mathrm{V}$ latency represents the sum of mechanical and neural components. Neely et al. (1988) assumed the neural component to be independent of frequency and level. The second term in their Eq. (1) was assumed to represent the component of ABR latency due to the mechanical propagation within the cochlea. Hence they estimated the BM group delay by

$$
\tau_{\mathrm{BM}, \mathrm{A}}(i, f)=b c^{-i} f^{-d},
$$

where $i$ represents the tone-burst intensity (in $\mathrm{dB}$ SPL divided by 100), $f$ represents tone-burst frequency (divided by $1 \mathrm{kHz}$ ), and $b, c$, and $d$ are constants with the values $b$ $=12.9 \mathrm{~ms}, c=5.0$, and $d=0.413$, according to the data fit from Neely et al. (1988). Whether this term actually represents only the mechanical component of the observed ABR latency, as assumed by Neely et al. (1988), or inherently also some frequency and/or level-dependent neural/synaptic contribution, has not been resolved yet. In any case, Eq. (8) represents the frequency and level-dependent part of wave- $\mathrm{V}$ latency. From this, the inverse function and the function for the instantaneous phase $\varphi_{\mathrm{A}}(i, t)$ can be calculated as follows: ${ }^{3}$

$\varphi_{\mathrm{A}}(i, t)=\frac{2 \pi\left(b c^{-i}\right)^{1 / d}}{\frac{1}{d}-1}\left[\frac{1}{\left(t_{0}(i)-t\right)^{1 / d-1}}-\frac{1}{t_{0}(i)^{1 / d-1}}\right]$.

The chirp stimulus is then given by

$$
s_{\mathrm{A}}(i, t)=A_{\mathrm{A}}(i, t) \sin \left[\varphi_{\mathrm{A}}(i, t)-\varphi_{0}\right],
$$

whereby $\varphi_{0}$ defines the starting phase of the chirp. The amplitude factor $A_{\mathrm{A}}(i, t)$ was chosen as

$$
A_{\mathrm{A}}(i, t)=\sqrt{\frac{\left(b c^{-i}\right)^{1 / d}}{d\left[t_{0}(i)-t\right]^{1 / d+1}}}
$$

in order to produce a stimulus with flat magnitude spectrum. Throughout the current study, the stimulus $s_{\mathrm{A}}(i, t)$ will be referred to as the "A-chirp," since it is based on ABR data.

\section{Comparison of the different chirp stimuli}

The O- and A-chirps are compared to the original chirp defined in Dau et al. (2000) that was based on de Boer's (1980) linear cochlea model. For direct comparison, the realization of the chirp with a flat magnitude spectrum is used that was also developed in Dau et al. (2000) and denoted as the "flat-spectrum chirp." Since this chirp is based on a model, it is referred to as the "M-chirp" in the following.

Within de Boer's (1980) model, the propagation time, $\tau_{\mathrm{BM}, \mathrm{M}}(f)$, needed to arrive at the place of resonance for the frequency $f$, is approximately given as

$$
\tau_{\mathrm{BM}, \mathrm{M}}(f) \propto(f+165.4 \mathrm{~Hz})^{-1.1},
$$

which clearly differs from Eqs. (2) and (8) representing the corresponding functions for the two other chirps. The left panel of Fig. 1 illustrates the calculated BM group delays on the basis of Eqs. (2), (8), and (12). The group delays derived from the SFOAE experiments (solid curve) are about 2-5 ms larger than those predicted on the basis of de Boer's model (dashed curve). The shaded dots represent the original BM group delay estimates of the SFOAE data by Shera and Guinan (2000). The shift of the SFOAE-based estimates to- 

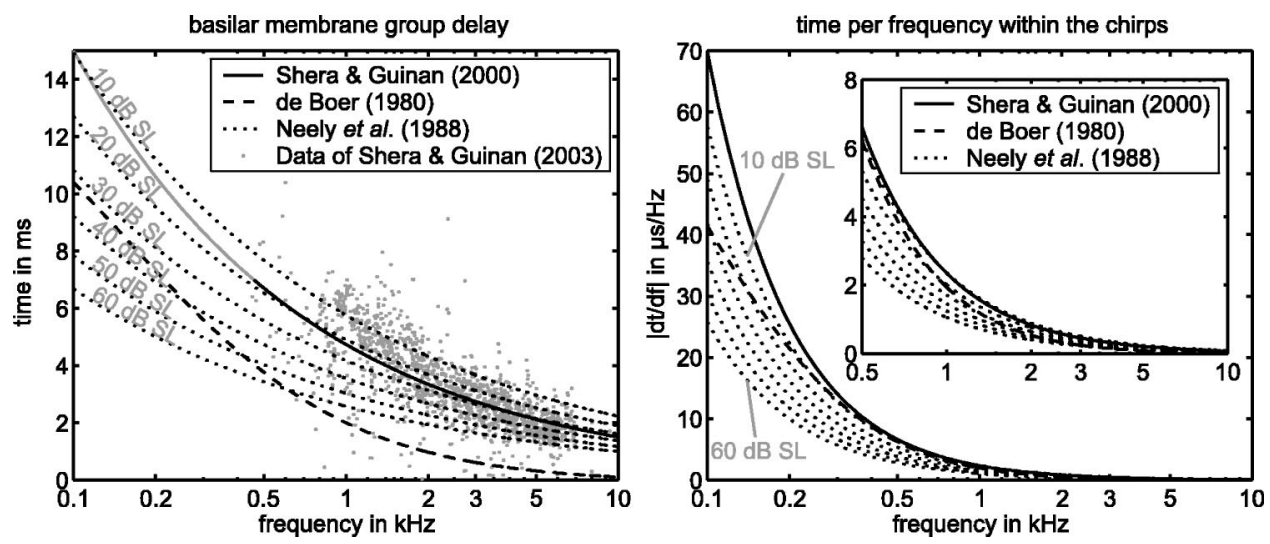

FIG. 1. Left: BM group delay as a function of frequency. The shaded dots represent the original BM group delay estimates of Shera and Guinan (2003), derived from SFOAE data. The black solid line represents the data fit of Shera and Guinan (2000). This function is interpolated toward the lower frequencies (gray solid line). The dashed line represents the group delay on the basis of the linear cochlea model by de Boer (1980). The dotted lines indicate the group delays predicted by Neely et al. (1988) for stimulus intensities from $10 \mathrm{~dB}$ SL (top dotted curve) up to $60 \mathrm{~dB}$ SL (bottom dotted curve). Right: Time-perfrequency change for the different chirps. This was directly calculated from the data in the left panel. The inset is a replot of the data for the frequency range from 0.5 to $10 \mathrm{kHz}$, using a rescaled ordinate.

ward higher values is reasonable, at least qualitatively, since frequency selectivity is increased at lower levels and this should be accompanied by a larger group delay at all frequencies. The group delay estimates predicted by the equation of Neely et al. (1988) depend on frequency and intensity (dotted curves). At medium levels, the delay is between about $1 \mathrm{~ms}$ (at $10 \mathrm{kHz}$ ) and about $8 \mathrm{~ms}$ (at $0.1 \mathrm{kHz}$ ).

For the generation of the chirps, not the absolute values but the change of group delay with frequency is important. Thus, the derivative of the group-delay versus frequency function is shown in the right panel of Fig. 1. The main differences between the SFOAE-based curve and the modelbased prediction occur at low frequencies, but differences are
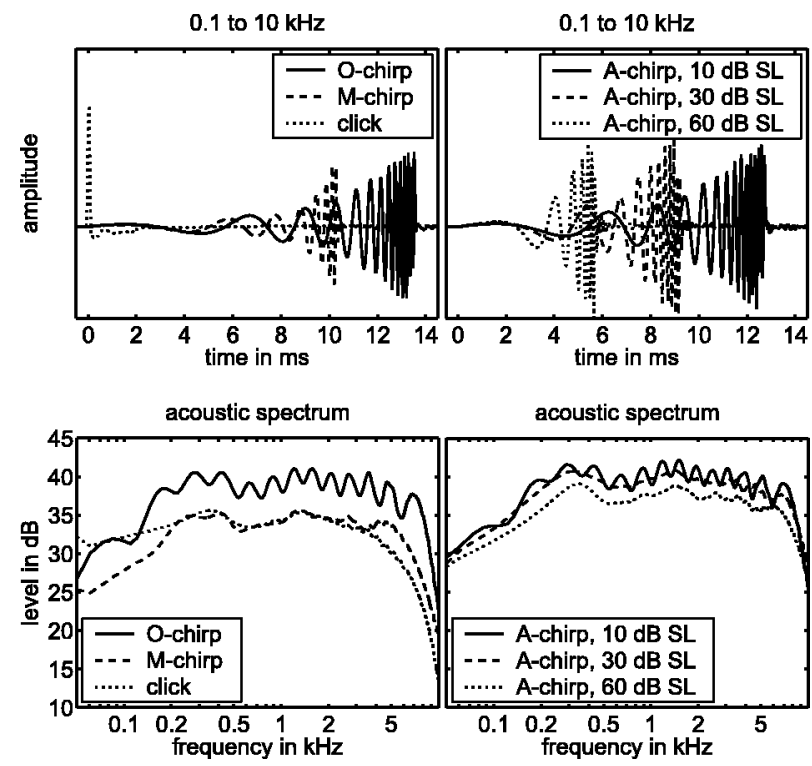

FIG. 2. Temporal waveforms (upper panel) and corresponding acoustic spectra (lower panel) of the broadband stimuli $(0.1-10 \mathrm{kHz})$. The left panels show the O-chirp, the M-chirp, and the click stimulus, which are indicated as solid, dashed, and dotted functions, respectively. The right panels show the corresponding functions for the level dependent A-chirps generated for 10, 30, and $60 \mathrm{~dB}$ SL. Different levels were indicated by different line styles. For better comparison, all waveforms are shown for a level of $100 \mathrm{~dB}$ peSPL. also present at frequencies above $500 \mathrm{~Hz}$. This means that the instantaneous frequency of the corresponding O-chirp will vary more slowly than that of the M-chirp, particularly at low frequencies. The corresponding ABR-based groupdelay functions clearly differ from the other ones. There is a large variation with stimulus level. For example, at higher levels, the resulting A-chirp will vary much faster in instantaneous frequency over time than in the cases of the O- and M-chirp, resulting in much shorter chirp durations. This is shown in Fig. 2 and described in more detail further below.

\section{METHOD}

\section{A. Subjects}

Nine normal-hearing subjects (two female and seven male) with no history of hearing problems and audiometric thresholds of $15 \mathrm{~dB}$ HL or better participated in the experiments. All subjects were between 28 and 38 years of age, and either volunteered or were paid for the participation in the experiments.

\section{B. Apparatus}

The experiments were carried out with a PC-based computer system which controlled stimulus presentation and recording of evoked potentials. A digital signal processing (DSP) card (Ariel DSP32C) converted the digitally generated stimulus (16 bit, $25 \mathrm{kHz}$ sampling rate) to an analogous waveform. The output of the DSP card was connected to a digitally controlled audiometric amplifier, which presented the stimulus through an insert earphone (Etymotic Research ER-2) to the subject.

Electroencephalic activity was recorded from the scalp via silver/silver chloride electrodes, attached to the vertex (positive) and the ipsilateral mastoid (negative). The forehead served as the site for the ground electrode. Interelectrode impedance was maintained below $5 \mathrm{k} \Omega$. Responses were amplified $(80 \mathrm{~dB})$ and filtered $(30-3000 \mathrm{~Hz})$ with a commercially available evoked potential amplifier (TDT DB4/HS4). The amplified signal was digitized by the 

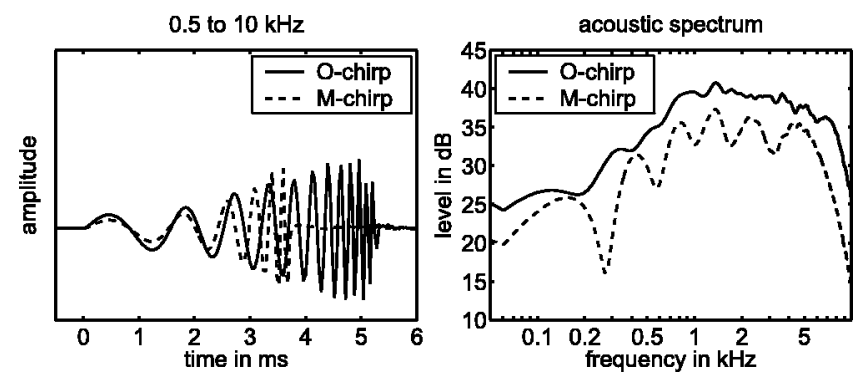

FIG. 3. Temporal waveforms (left panel) and corresponding acoustic spectra (right panel) of the $(0.5-10-\mathrm{kHz}) \mathrm{M}$-chirp (dashed lines) and the (0.5-10$\mathrm{kHz}$ ) O-chirp (solid lines).

DSP card (16 bit, $25 \mathrm{kHz}$ sampling rate), which also performed artifact rejection and signal averaging. Responses were recorded for $37 \mathrm{~ms}$ following the stimulus onset. Offline filtering (digital low-pass, $1600 \mathrm{~Hz}$, order 4) was done to suppress noise.

\section{Stimuli and procedure}

Chirps as described in Sec. II were used as stimuli. The nominal edge frequencies of the chirps were 0.1 and $10 \mathrm{kHz}$ resulting in durations of $13.52 \mathrm{~ms}$ for the O-chirp and 10.32 ms for the M-chirp. The durations for the A-chirp varied between $5.72 \mathrm{~ms}$ for a sensation level (SL) of $60 \mathrm{~dB}$ and $12.72 \mathrm{~ms}$ for $10 \mathrm{~dB}$ SL. To compare results with standard ABR measurements, an $80-\mu$ s click stimulus was generated. The upper left panel of Fig. 2 shows the acoustic waveforms of the O-chirp, the M-chirp, and the click stimulus. The waveforms of the A-chirp for stimulation levels of 10, 30, and $60 \mathrm{~dB}$ SL are depicted in the upper right panel. The corresponding acoustic spectra are given in the lower panels. They were obtained by coupling the ER-2 insert earphone to a Brüel and Kjær ear simulator (type 4157) with a 1/2-inch condenser microphone (type 4134), a 2669 preamplifier, and a 2610 measuring amplifier. The spectra were derived from fast Fourier transforms (FFTs) of 100-trial time-domain averages of the stimulus over an analysis frame of 2048 samples using a sampling rate of $25 \mathrm{kHz}$. The waveforms were not windowed prior to FFT.

Since Shera and Guinan (2000) collected only very few data points below $0.5 \mathrm{kHz}$, Eq. (2) may represent only a poor description of the real behavior in this frequency region. Therefore, additional O- and M-chirp stimuli were generated with nominal edge frequencies of 0.5 and $10 \mathrm{kHz}$. The corresponding durations were $5.24 \mathrm{~ms}$ for the O-chirp and 3.68 $\mathrm{ms}$ for the M-chirp. Figure 3 shows the acoustic waveforms (left panel) and spectra (right panel) of these stimuli.

For all stimuli, the presentation level was varied between 10 and $60 \mathrm{~dB}$ SL in 10-dB steps. To determine the sensation level for the click, M-chirp and O-chirp, the absolute hearing thresholds were measured individually with an adaptive three-interval three-alternative forced-choice (oneup, two-down) procedure. At threshold (0 dB SL) the mean peak-equivalent sound pressure level (peSPL) was $47.2 \mathrm{~dB}$ for the click, $40.5 \mathrm{~dB}$ for the $0.1-10-\mathrm{kHz}$ M-chirp, and 37.3 $\mathrm{dB}$ for the $0.1-10-\mathrm{kHz}$ O-chirp.
The time course of the A-chirp varies with level. Thus, to be able to calculate the time course of, e.g., the 60-dB SL A-chirp one needs to know the peSPL value corresponding to $60 \mathrm{~dB}$ SL. This requires the knowledge of the corresponding hearing threshold. To determine the hearing threshold one needs to have the time course of the corresponding chirp. To get around this problem, a hearing threshold of $40 \mathrm{~dB}$ peSPL was assumed, comparable to the one for the M-chirp. This resulted in presentation levels between 50 and $100 \mathrm{~dB}$ peSPL, corresponding to the sensation levels from 10 to 60 $\mathrm{dB}$. To verify this approach, hearing thresholds for the 10-, 30- and 60-dB SL realizations of the A-chirp were measured individually with the same procedure as described above. This resulted in different hearing thresholds for the different chirps, with a mean value of $37.5 \mathrm{~dB}$ peSPL, ranging from 36.1 to $38.9 \mathrm{~dB}$ peSPL. The presentation levels for the A-chirp were therefore overestimated by less then $3.9 \mathrm{~dB}^{4}$

The subject lay on a couch in an electrically shielded, soundproof room, and electrodes were attached. The subject was instructed to keep movement at a minimum, and to sleep if possible. The lights were turned off at the beginning of the session. Each session lasted between one and two hours, depending on the subject's ability to remain still. The ear of stimulation was chosen randomly, i.e., for each subject one ear was chosen and then maintained. The acoustic signals were delivered at a repetition rate of $20 \mathrm{~Hz}$ for all stimulus conditions. A temporal jitter of $\pm 2 \mathrm{~ms}$ was introduced to minimize response superimposition from preceding stimuli. Thus the time interval between the onsets of two successive stimuli varied randomly and equally distributed between 48 and $52 \mathrm{~ms}$. Each trial consisted of 3000 averages. For each stimulus condition, two independent trials were stored in separate buffers. These are illustrated as superimposed wave forms in the figures to show response replicability.

\section{Statistical analysis}

Wave-V peak-to-peak amplitude was analyzed in all stimulus conditions. The amplitude was measured from the peak to the largest negativity following it. For each condition, wave- $\mathrm{V}$ amplitude was averaged across subjects. A Wilcoxon matched-pairs signed-rank test $(\alpha=0.05)$ was performed to test whether the response amplitude differed significantly for two comparison stimuli.

\section{RESULTS}

Figure 4 shows mean $A B R$, averaged across all nine subjects, obtained with the OAE-based (0.1-10-kHz) O-chirp (upper left panel), the original model-based M-chirp (upper right panel), the ABR-based A-chirp (lower left panel), and the click (lower right panel). Results for different stimulus levels are indicated on separate axes along the ordinate, and labeled with the corresponding sensation level (dB SL). Wave- $\mathrm{V}$ peaks are marked by small black triangles. Wave $\mathrm{V}$ is the only peak that can be observed in all stimulus conditions. For the O-chirp, no earlier waves are present, even at the highest stimulation levels. In contrast, for the M-chirp, 


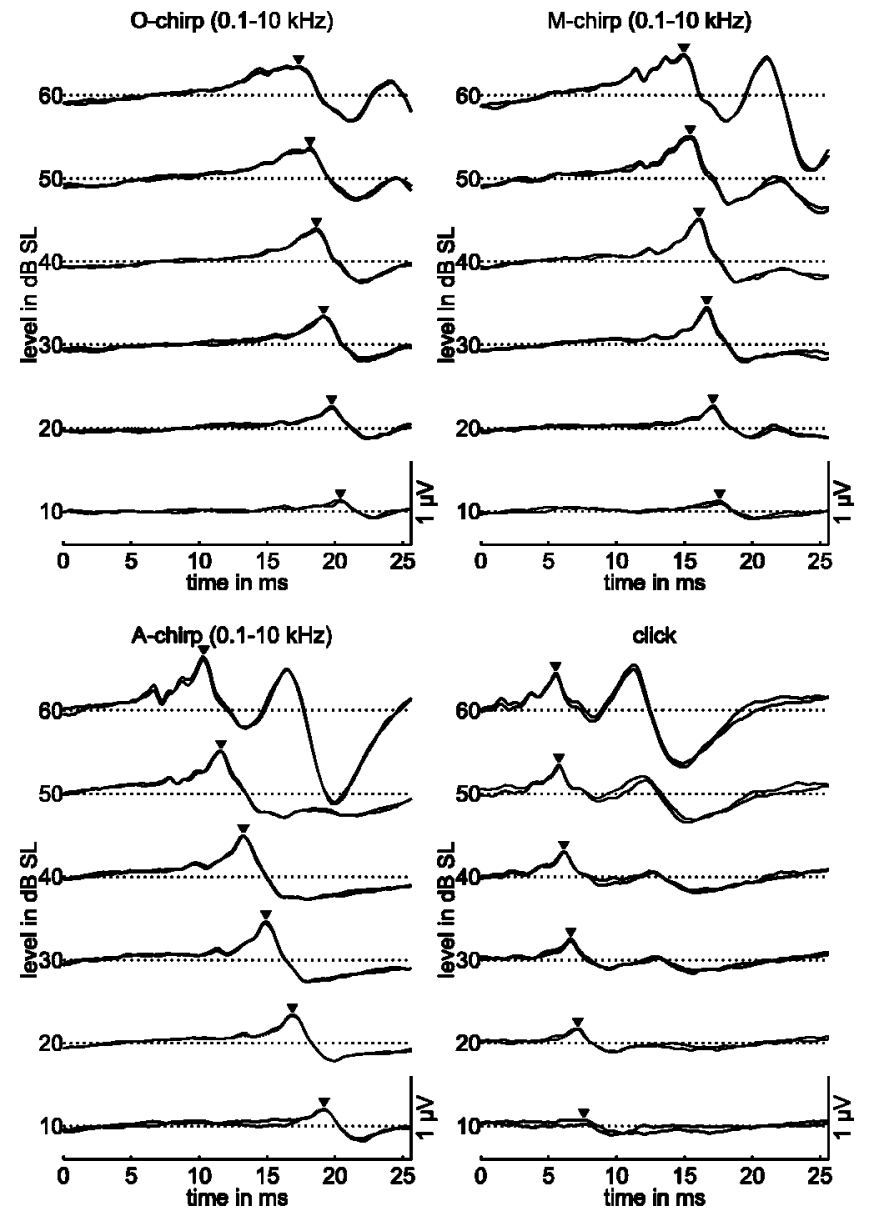

FIG. 4. ABR waveforms obtained with the $0.1-10-\mathrm{kHz}$ O-chirp (upper left panel), M-chirp (upper right panel), A-chirp (lower left panel), and click (lower right panel), averaged across all nine subjects. The stimulation level varied from 10 to $60 \mathrm{~dB}$ SL, as indicated. At each level, two independently averaged waveforms are superimposed to show response replicability. The black triangles indicate wave- $\mathrm{V}$ peaks.

A-chirp, and the click, waves I and III become visible at the highest levels. Interestingly, for the A-chirp, wave I is visible even down to a level of $20 \mathrm{~dB}$ SL.

Figure 5 (left panel) summarizes the quantitative values for the mean wave- $\mathrm{V}$ amplitude as a function of the stimulation level. The click-evoked wave- $\mathrm{V}$ amplitude, represented by the filled squares, is always smaller than that obtained with any of the broadband chirps, represented by the other filled symbols. For example, the M-chirp (filled circles), leads to amplitude values that are more than twice the values for the click at most stimulus levels. This agrees well with the results found in Dau et al. (2000). At the lowest stimulation level, the A-chirp (filled downward triangles) evoked an amplitude that is about three times as large as that for the click. The amplitude-level function for the A-chirp looks like shifted by about $0.44 \mu \mathrm{V}$ with respect to the click curve. For the A- and M-chirp, statistical analysis revealed significantly larger amplitudes than for the click at all stimulus levels while for the (broadband) O-chirp the difference was significant only for 10 and $40 \mathrm{~dB}$ SL.

Now consider the results for the O-chirp (filled upward triangles) in comparison to the original M-chirp (filled circles), having in mind that the O-chirp was based on $40 \mathrm{~dB}$ SPL otoacoustic emission data while the M-chirp was derived from a (linear) cochlea model based on high-level BM data. At levels of $40 \mathrm{~dB}$ SL and above, wave- $\mathrm{V}$ amplitude is smaller for the O-chirp than for the M-chirp, while at the lower levels, wave- $\mathrm{V}$ amplitude is about the same for the two stimuli. Statistical analysis of the amplitude data revealed significant differences between the $\mathrm{O}$ - and M-chirp only for levels of 50 and $60 \mathrm{~dB}$ SL, where wave-V amplitude for the M-chirp is higher. The results for the smaller chirp bandwidth, ranging from $0.5-10 \mathrm{kHz}$, are indicated by the corresponding open symbols. Results for the O- and the M-chirp are given by triangles and circles, respectively. The response waveforms are not shown explicitly for these two conditions. Statistical analysis revealed a significant difference between the two chirps only for a level of $20 \mathrm{~dB}$ SL, where the O-chirp evoked a higher wave-V amplitude than the M-chirp.

Next consider the results for the A-chirp (filled downward triangles) in comparison to the original one (M-chirp). The A-chirp resulted in a larger wave-V amplitude than the M-chirp (and any other stimulus tested here) at nearly all stimulation levels. However, statistical analysis revealed significant differences between A- and M-chirp only for low stimulation levels (10 and $20 \mathrm{~dB}$ SL).

Comparison of the results for the A-chirp and the O-chirp shows that the A-chirp elicited a higher wave-V amplitude than the O-chirp at all stimulation levels used here. In this case, statistical analysis results in significant differences for low and high stimulation levels $(10,20,50$, and $60 \mathrm{~dB}$ SL).
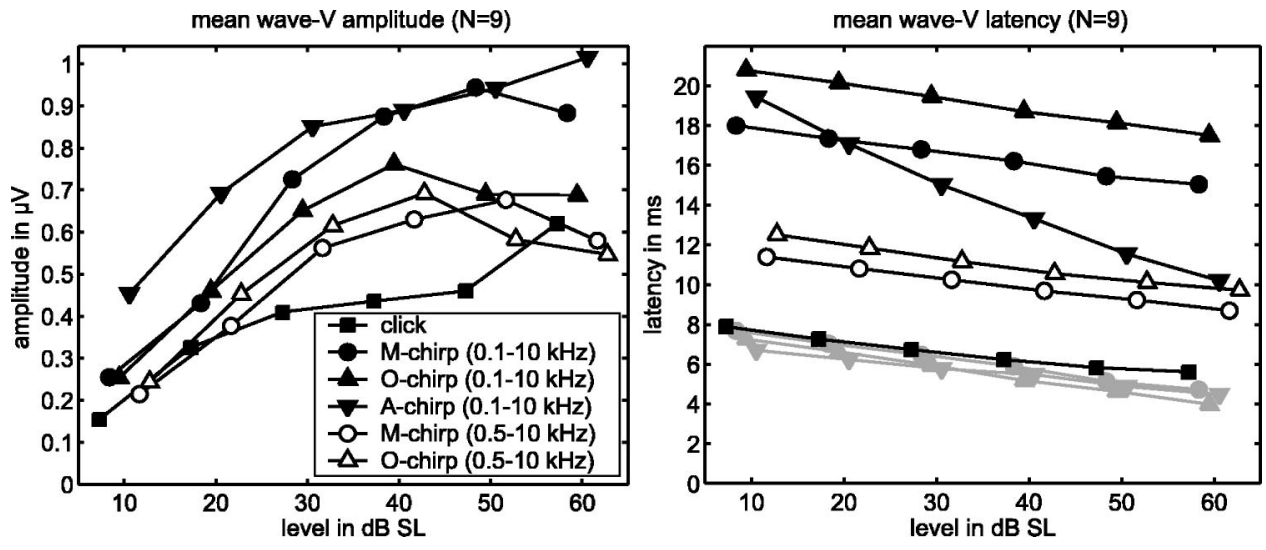

FIG. 5. Average ABR wave-V amplitude (left panel) and latency (right panel), as a function of stimulation level. Different symbols indicate different stimulus conditions. $\mathbf{\square}$ : click, ○: $0.1-10-\mathrm{kHz}$ M-chirp, $\mathbf{\Delta}: 0.1-10-$ kHz O-chirp, $\mathbf{\nabla}$ : 0.1-10-kHz A-chirp, $\bigcirc: 0.5-10-\mathrm{kHz}$ M-chirp, and $\triangle: 0.5-$ 10-kHz O-chirp. The shaded symbols in the right panel indicate the offset latencies for the corresponding stimuli. For better visibility, the symbols are slightly shifted along the abscissa. 
The right panel of Fig. 5 shows the mean wave-V latency behavior obtained with the different stimuli. Except for the A-chirp, all functions are roughly in parallel to each other but shifted relative to each other by some amount. For these functions, the latency decreases by about $2-3 \mathrm{~ms}$ for a $50-\mathrm{dB}$ level change (from 60 to $10 \mathrm{~dB} \mathrm{SL}$ ), which is consistent with literature data (e.g., Hoth and Lenarz, 1994). The main differences between the functions correspond to the differences in the respective stimulus durations, as is illustrated by the shaded functions in the same panel of the figure. They indicate the latency values for the three broadband chirps relative to stimulus offset instead of stimulus onset. The very similar values in this view are consistent with the idea behind the chirp paradigm that, ideally, the displacement maxima on the BM should occur in all channels at the same time, and thus, the latencies for the chirp and the click should be similar if expressed relative to stimulus offset. Thus, since the duration of the A-chirp changes strongly with level, this must be directly reflected in a relatively steep function if expressed relative to stimulus onset.

\section{DISCUSSION}

Previous studies have shown that an upward chirp can evoke a significantly larger wave- $\mathrm{V}$ amplitude than the conventional click (Dau et al., 2000; Wegner and Dau, 2002). The equations defining the upward chirp in these earlier studies were calculated to be the inverse of the delay-line characteristic of the cochlear partition on the basis of de Boer's (1980) cochlea model. However, since this model does not take BM nonlinearity (compression) into account which is associated with level-dependent frequency selectivity, the model probably underestimates real BM group delays at low and medium stimulus intensities. The intention of the present study was to design and test chirp stimuli that might potentially cause an even larger neural synchronization than the original chirp, at least at lower stimulus intensities. Two different strategies for the generation of the stimuli were used: One stimulus, the O-chirp, was calculated from stimulusfrequency-emission group-delay data by Shera and Guinan (2000), recorded at a stimulation level of $40 \mathrm{~dB}$ SPL in humans. The other stimulus, the A-chirp, was based on measured $A B R$ wave- $V$ latency values obtained with tone pulses at various frequencies and levels. The corresponding responses evoked by these chirps were compared with results obtained with the original M-chirp and the click.

\section{A. Usefulness of the OAE-based chirp stimulation for ABR}

The data of the present study showed only small differences between $\mathrm{O}-$ and M-chirp stimulation. For the frequency region above $500 \mathrm{~Hz}$, where reliable SFOAE data were available, the two $0.5-10-\mathrm{kHz}$ chirps produced about the same wave- $\mathrm{V}$ amplitude (open symbols in Fig. 5). No significant advantage was found for the O-chirp at the lower levels (except for $20 \mathrm{~dB} \mathrm{SL}$ ), and no advantage was observed for the M-chirp at higher levels. For the broadband conditions $(0.1-10 \mathrm{kHz}$; closed circles and upward triangles in Fig. 5), the M-chirp produced a larger potential amplitude than the corresponding O-chirp at the two highest levels.

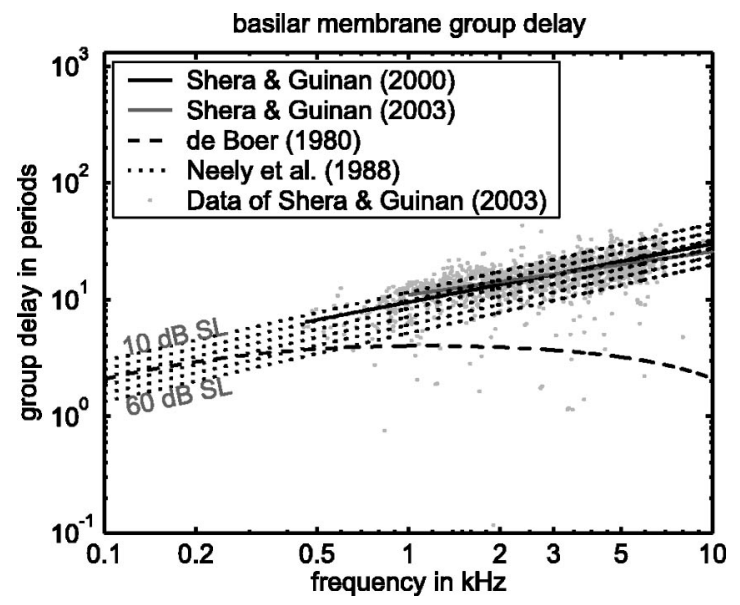

FIG. 6. BM group delay as a function of frequency. The group delay is indicated in dimensionless form in units of periods of the stimulus frequency. The shaded dots represent the BM group delay estimates of Shera and Guinan (2003), derived from SFOAE data. The gray solid line (starting at $1 \mathrm{kHz}$ ) represents their recent data fit, while the black solid line represents the fit function from 2000. The latter one was used in the present study as the basis for the generation of the O-chirp. The dashed line indicates the group delay based on the cochlea model by de Boer (1980). The dotted lines show the group delays predicted from Neely et al. (1988) for stimulus intensities ranging from $10 \mathrm{~dB}$ SL (top dotted curve) up to $60 \mathrm{~dB}$ SL (bottom dotted curve).

This indicates that, for the O-chirp, our assumed extrapolated group-delay-versus-frequency function at the very low frequencies $(0.1-0.5 \mathrm{kHz})$ probably does not match the real system very well. At these very low frequencies, the function underlying the M-chirp appears to represent the better choice.

What might be the reason(s) for the similar results at low intensities obtained with these two stimuli? The hypothesis underlying the generation of the O-chirp was that, according to the theory of reflection filtering (Shera and Zweig, 1993; Zweig and Shera, 1995), reflection-source emission group delay is determined by the group delay of the BM mechanical transfer function at its peak. Thus, otoacoustic emissions may be used to provide a noninvasive measure of BM group delay, at least at low levels. However, it is possible that the estimate of the BM group delay by the SFOAEbased group delay is not very reliable. First, the emission data show large scatter and vary considerably with frequency (see Fig. 1), even though the trend lines of Shera and Guinan (2003) were quite robust within and across subjects. This large variability seems consistent with results from the study by Neely et al. (1988) where also large variations of OAE data were found within and across subjects. The scatter in the OAE data does not seem to arise from measurement noisethe measurements are quite reproducible in each subject (Shera and Guinan, 2003). Instead, the scatter may come from intrinsic variations in emission phase that are correlated with variations in emission amplitude across frequency (Shera and Guinan, 2003). Second, the ratio $\tau_{\mathrm{SFOAE}} / \tau_{\mathrm{BM}}$ (derived from their animal experiments) was found to be somewhat less than the predicted value of 2 such that there might be a mismatch in the predicted BM group delay above 1 $\mathrm{kHz}$. Third, further following the recent findings of Shera and Guinan (2003), there is a breakdown in the proportion- 
ality between emission and mechanical BM group delay in the apical part of the cochlea. Thus, for frequencies below 1 $\mathrm{kHz}$, it is uncertain if the SFOAE data can be used to get a reliable estimate of BM group delay.

The O-chirp of the present study was based on a formula whose parameters differ somewhat from those suggested by Shera and Guinan on the basis of their most recent results (Shera and Guinan, 2003). The group-delay versus frequency function representing their fit to these recent data is indicated as the gray line starting at $1 \mathrm{kHz}$ in Fig. 6 . The group delay in this figure is indicated in dimensionless form in units of periods of the stimulus frequency, and expressed as BM group delay [assuming $\left.\tau_{\mathrm{BM}}(f)=0.5 \times \tau_{\mathrm{SFOAE}}(f)\right]$. In addition, the raw data as well as the other curves from Fig. 1 are replotted here for comparison. The different scale was used in order to make the data easily comparable with the figures in Shera and Guinan (2003). It is possible, in principle, that a chirp based on the estimate, in combination with an appropriate extension toward the lower frequencies $(<1 \mathrm{kHz})$, would lead to larger evoked response amplitudes.

Indeed, the finding that the OAE based chirp does not produce a larger response amplitude than the other chirps does not necessarily imply that SFOAE group delay is a poor estimator of BM group delay. The contributions of the lowfrequency components of the stimulus to the $\mathrm{ABR}$ are mainly responsible for the advantage of the chirp over the click (Dau et al., 2000; Wegner and Dau, 2002). In fact, it is still possible that the estimate of BM group delay using SFOAE is reasonable at medium and high frequencies while it may be problematic at frequencies below about $1 \mathrm{kHz}$. Another aspect is that it is problematic to compare the results of the intensity-independent O-chirp with the results of the intensity-dependent A-chirp except at levels near the one used in the OAE measurements (40 dB SPL). It is possible, at least in principle, that had the SFOAE delay estimates been available for a similar range of intensities, no significant differences between A- and O-chirps would have been found at any intensity. At 30 and $40 \mathrm{~dB}$ SL, the differences were not significant (see Sec. IV). Finally, in more general terms, it is important to note, that a stimulus that causes a maximum amount of synchronicity at brainstem level (where wave $\mathrm{V}$ is generated) does not necessarily imply that the same stimulus also causes a maximum synchronized activity at BM level, as has been implicitly assumed in our previous studies (Dau et al., 2000; Wegner and Dau, 2002) and also indirectly in the study by Neely et al. (1988). Wave-V latency always represents the sum of a mechanical and a neural delay, and the neural delay also might be frequency- and level-dependent. It is not well known what exactly needs to be synchronized to maximize ABR amplitude. It is possible that maximal $A B R$ occur when first-spike latencies are equalized across CF. However, first-spike latencies are not necessarily corresponding to group delays. Thus, the O-chirp might not be optimal even if SFOAEs would provide a good measure of BM group delay.

\section{B. Capabilities of the ABR-based chirp-stimulation paradigm}

The recordings obtained with the A-chirps showed the largest response amplitude. Indeed, wave- $\mathrm{V}$ amplitudes for the A-chirp were found to be higher than for any of the other stimuli of the present study at most stimulation levels. This was true even though the chirp was designed on the basis of average data from a completely different set of subjects (Gorga et al., 1988) and using different equipment for the recordings as in the present study. One might argue that it is not surprising to obtain good ABR results with a stimulus that was developed on the basis of ABR data. On the other hand, for a complex and nonlinear system like the auditory system, it is not clear in advance that a composite stimulus like the chirp, that sweeps through the frequencies at a rate determined by the latency values obtained in separate recordings with (transient) tone pulses would necessarily lead to such large responses. At medium and high stimulation levels, the excitation on the BM in response to tone pulses is certainly not frequency specific, due to effects of spread of excitation associated with cochlear nonlinearities. However, at low levels, the quasilinear approach that is implicitly assumed in the chirp-generation paradigm might be appropriate. The results of the present study clearly suggest that the A-chirp represents a very effective stimulus. The advantage is particularly large at low stimulation levels where the response amplitude is about three times as large as that obtained with click stimulation (even though the peak equivalent sound pressure level of the click was about $10 \mathrm{~dB}$ higher than that for the A-chirp if compared at the same SL). The finding that the A- chirp also produces the clearest wave I suggests that it also very effectively stimulates the earliest neural processing station, the auditory nerve. However, since the derivation of the chirp was based on ABR wave-V latency data, it remains not possible to clearly separate between mechanical and neural/synaptic delays. In fact, the results of the present paper do not depend on whether the assumptions of Neely et al. (1988) about the relative contributions of mechanical and neural delay are true or not. It is not possible to finally verify or falsify their assumptions on the basis of our experimental data. However, whatever the exact contributions of the different components to the overall delay are, the A-chirp may in any case be interesting and valuable for clinical application, e.g., as an objective indicator of hearing threshold. It might be particularly useful in all applications where the traditional click stimulus has been used so far.

\section{SUMMARY AND CONCLUSIONS}

- Two chirp stimuli were developed such as to compensate for travel-time differences across frequency. One stimulus, the O-chirp, was based on BM group-delay estimates (at $40 \mathrm{~dB}$ SPL) obtained with SFOAE (Shera and Guinan, 2000). The other one, the A-chirp, was based on functions fitted to tone-pulse-evoked ABR wave- $\mathrm{V}$ latencies at various stimulation levels (Gorga et al., 1988; Neely et al., 1988). ABR obtained with these chirps were compared to 
click responses and responses to the original chirp stimulus, the M-chirp, which is based on a linear cochlea model (Dau et al., 2000).

- All chirps caused a larger wave-V response amplitude than the click. This is mainly caused by activity from the entire frequency range contributing to the chirp response while in the case of the click the lower frequencies do not contribute effectively to the response.

- No significant differences between the response amplitudes obtained with the O-chirp and the M-chirp were found, not even at low stimulation levels where an advantage of the O-chirp was expected. One possible explanation might be that SFOAE group delays do not allow a reliable estimate of BM group delays, particularly at low frequencies $(<1 \mathrm{kHz})$. Another explanation might be that level- and frequency-dependent neural delays are involved in ABR (wave-V) latency which are not reflected in the design of these two chirps.

- The A-chirp caused the largest responses and is particularly effective at very low levels where wave-V amplitude is about three times as large as for the click. This leveldependent chirp intrinsically includes both mechanical and neural delays since it was derived from wave-V latency data. The A-chirp might be very useful for clinical applications, e.g., in connection with objective tests of hearing threshold. Specifically, this chirp might be valuable in all applications where the standard click stimulus has been used so far.

\section{ACKNOWLEDGMENTS}

The authors would like to thank the Associate Editor and two anonymous reviewers for their constructive and helpful comments and suggestions. The authors thank Chris Shera for very interesting discussions and for providing us with his data. The authors also thank our colleagues from the research group "Medizinische Physik" at the University of Oldenburg for fruitful discussions. This study was supported by the Deutsche Forschungsgemeinschaft (DFG), Research Program "Neural basis of cognitive functions" (SFB 517). Finally, the authors are grateful for the support from the Technical University of Denmark (DTU) in the final phase of this project.

${ }^{1}$ These constants describe the fit to the data of Shera and Guinan (2000). In the meantime, the authors collected more data points and provided different values for the two parameters: $c=0.43 \mathrm{~s}$ and $\alpha=-0.63$ (Shera and Guinan 2003).

${ }^{2}$ The time point $t_{0}$ can be chosen somewhat arbitrarily. The only constraint is that the denominators of the fractions inside the brackets in Eq. (5) are not allowed to be zero. Dau et al. (2000) used $t_{0}=t(0 \mathrm{~Hz})$. Since this value is not defined within Eq. (2), we used $t_{0}=\tau_{\mathrm{BM}, \mathrm{O}}(50 \mathrm{~Hz})$.

${ }^{3} t_{0}(i)$ can again be chosen somewhat arbitrarily, but in this case it is a function of the stimulus intensity $i$. We chose $t_{0}(i)=\tau_{\mathrm{BM}, \mathrm{A}}(i, 50 \mathrm{~Hz})$.

${ }^{4} \mathrm{~A}$ more accurate method would have been to use these thresholds to calculate new realizations of the 10- to 60-dB SL A-chirps and to measure their thresholds again. However, this would have resulted in a time-consuming iterative process.

von Békésy, G. (1960). Experiments in Hearing (McGraw-Hill, New York). de Boer, E. (1980). "Auditory physics. Physical principles in hearing theory I,” Phys. Rep. 62, 87-174.
Dau, T., Wagner, O., Mellert, V., and Kollmeier, B. (2000). “Auditory brainstem responses with optimized chirp signals compensating basilarmembrane dispersion," J. Acoust. Soc. Am. 107(3), 1530-1540.

Don, M., and Eggermont, J. J. (1978). "Analysis of the click-evoked brain stem potentials in man using high-pass noise masking," J. Acoust. Soc. Am. 63(4), 1084-1092.

Don, M., Ponton, C. W., Eggermont, J. J., and Masuda, A. (1994). “Auditory brainstem response (ABR) peak amplitude variability reflects individual differences in cochlear response times," J. Acoust. Soc. Am. 96(6), 3476-3491.

Don, M., Masuda, A., Nelson, R., and Brackmann, D. (1997). "Successful detection of small acoustic tumors using the stacked derived-band auditory brain stem response amplitude," Am. J. Otol. 18(5), 608-621.

Gorga, M. P., Kaminski, J. R., Beauchaine, K. A., and Jestead, W. (1988). "Auditory brain-stem responses to tone bursts in normal hearing subjects,” J. Speech Hear. Res. 31(1), 87-97.

Greenwood, D. D. (1990). "A cochlear frequency position function for several species-29 years later," J. Acoust. Soc. Am. 87(6), 2592-2605.

Hoth, S. and Lenarz, T. (1994). Elektrische Reaktionsaudiometrie (Springer, Berlin).

Kiang, N. Y. S. (1975). "Stimulus representation in the discharge patterns of auditory neurons," in The Nervous System, Vol. 3: Human Communication and Its Disorders, edited by D.B. Tower (Raven, New York), pp. 81-95.

Kiang, N. Y. S., Watanabe, T., Thomas, E. C., and Clark, L. F. (1965). Discharge Patterns of Single Fibers in the Cat's Auditory Nerve M.I.T. Research Monographs 35 (MIT Press, Cambridge).

Moore, B. C., and Glasberg, B. R. (1997). "A model of loudness perception applied to cochlear hearing loss," Aud. Neurosci. 3, 289-311.

Moore, B. C. J., Vickers, D. A., Plack, C. J., and Oxenham, A. J. (1999). "Inter-relationship between different psychoacoustic measures assumed to be related to the cochlear active mechanism," J. Acoust. Soc. Am. 106(5), 2761-2778.

Neely, S. T., Norton, S. J., Gorga, M. P., and Jesteadt, W. (1988). "Latency of auditory brain-stem responses and otoacoustic emissions using toneburst stimuli," J. Acoust. Soc. Am. 83(2), 652-656.

Norton, S. J., and Neely, S. T. (1987). "Tone-burst-evoked oto-acoustic emissions in normal hearing subjects," J. Acoust. Soc. Am. 81(6), 18601872

Oxenham, A. J., and Plack, C. J. (1998). "Suppression and the upward spread of masking," J. Acoust. Soc. Am. 104(6), 3500-3510.

Oxenham, A. J., and Shera, C. A. (2003). "Estimates of Human Cochlear Tuning at Low Levels Using Forward and Simultaneous Masking," J. Assoc. Res. Otolaryngol. 4(4) in press.

Oxenham, A. J., Moore, B. C. J., and Vickers, D. A. (1997). "Short-term temporal integration: evidence for the influence of peripheral compression," J. Acoust. Soc. Am. 101(6), 3676-3687.

Plack, C. J., and Oxenham, A. J. (1998). "Basilar-membrane nonlinearity and the growth of forward masking," J. Acoust. Soc. Am. 103(3), 15981608.

Rhode, W. S. (1971). "Observations of the vibration of the basilar membrane in squirrel monkeys using the Mössbauer technique," J. Acoust. Soc. Am. 49(4), 1218-1231.

Rhode, W. S., and Recio, A. (2000). "Study of the mechanical motions in the basal region of the chinchilla cochlea," J. Acoust. Soc. Am. 107(6), 3317-3332.

Robles, L., Ruggero, M. A., and Rich, N. C. (1986). "Basilar membrane mechanics at the base of the chinchilla cochlea. I. Input-output functions, tuning curves, and response phases," J. Acoust. Soc. Am. 80(5), 13641374.

Ruggero, M. A., and Rich, N. C. (1991). "Furosemide alters organ of corti mechanics: evidence for feedback of outer hair cells upon the basilar membrane," J. Neurosci. 11(4), 1057-1067.

Ruggero, M. A., Rich, N. C., Robles, L., and Recio, A. (1995). "The effects of acoustic overstimulation, other cochlear injury and death on basilar membrane responses to sound," in Effects of Noise on Hearing: Vth International Symposium, edited by R.J. Salvi, A. Axelsson, D. Henderson, and R. Hamernik (Thieme Medical, Stockholm).

Ruggero, M. A., Rich, N. C., Recio, A., Narayan, S. S., and Robles, L. (1997). "Basilar-membrane responses to tones at the base of the chinchilla cochlea," J. Acoust. Soc. Am. 101(4), 2151-2163.

Sellick, P. M., Patuzzi, R., and Johnstone, B. M. (1982). "Measurement of basilar membrane motion in the guinea pig using the Mössbauer technique," J. Acoust. Soc. Am. 72(1), 131-141.

Shera, C. A., and Guinan, J. J. (2000). "Frequency dependence of stimulus- 
frequency-emission phase: Implications for cochlear mechanics," in Recent Developments in Auditory Mechanics, edited by H. Wada, T. Takasaka, K. Ikeda, K. Ohyama, and T. Koike (World Scientific, Singapore), pp. 381-387.

Shera, C. A., and Guinan, J. J. (2003). "Stimulus-frequency-emission group delay: A test of coherent reflection filtering and a window on cochlear tuning," J. Acoust. Soc. Am. 113(5), 2762-2772.

Shera, C. A., and Zweig, G. (1993), "Order from the chaos: Resolving the paradox of periodicity in evoked otoacoustic emission," in Biophysics of Hair-Cell Sensory Systems, edited by H. Duifhuis, J.W. Horst, P. van Dijk, and S.M. van Netten (World Scientific, Singapore), pp. 54-63.
Shera, C. A., Guinan, J. J., and Oxenham, A. J. (2002). "Revised estimates of human cochlear tuning from otoacoustic and behavioral measurements," Proc. Natl. Acad. Sci. U.S.A. 99(5), 3318-3323.

Wegner, O., and Dau, T. (2002). "Frequency specificity of chirp-evoked auditory brainstem responses," J. Acoust. Soc. Am. 111(3), 1318-1329.

Yates, G. K., Winter, I. M., and Robertson, D. (1990). "BM nonlinearity determines auditory nerve rate-intensity functions and cochlear dynamic range," Hear. Res. 45(3), 203-220.

Zweig, G. (1976). "Basilar membrane motion," Cold Spring Harbor Symp. Quant. Biol. 40, 619-633.

Zweig, G., and Shera, C. A. (1995). "The origin of periodicity in the spectrum of otoacoustic emissions," J. Acoust. Soc. Am. 98(4), 2018-2047. 